Published in final edited form as:

N Engl J Med. 2013 October 31; 369(18): 1715-1725. doi:10.1056/NEJMsa1214720.

\title{
Cost-Effectiveness of HIV Treatment as Prevention in Serodiscordant Couples
}

\author{
Rochelle P. Walensky, M.D., M.P.H. \\ Divisions of Infectious Disease and General Medicine, Medical Practice Evaluation Center, \\ Department of Medicine, Massachusetts General Hospital, Division of Infectious Disease, \\ Brigham and Women's Hospital, Harvard University Center for AIDS Research, Harvard Medical \\ School, Boston
}

Eric L. Ross, B.A.

General Medicine, Medical Practice Evaluation Center, Boston

Nagalingeswaran Kumarasamy, M.B., B.S., Ph.D.

Y.R. Gaitonde Center for AIDS Research and Education, Chennai, India

Robin Wood, D.Sc.

Desmond Tutu HIV Centre, Institute of Infectious Disease and Molecular Medicine, and Department of Medicine, University of Cape Town, Cape Town, South Africa

Farzad Noubary, Ph.D.

General Medicine, Medical Practice Evaluation Center, Boston

\section{A. David Paltiel, Ph.D., M.B.A.}

Yale School of Public Health, New Haven, CT

Yoriko M. Nakamura, B.A.

General Medicine, Medical Practice Evaluation Center, Boston

Sheela V. Godbole, M.D.

National AIDS Research Institute, Pune, India

Ravindre Panchia, M.B., B.Ch.

Perinatal HIV Research Unit

Ian Sanne, M.B., B.Ch., D.T.M.\&H.

Clinical HIV Research Unit

Milton C. Weinstein, Ph.D.

Department of Health Policy and Management, Boston

Elena Losina, Ph.D.

General Medicine, Medical Practice Evaluation Center, Department of Orthopaedic Surgery

Brigham and Women's Hospital, Harvard University Center for AIDS Research, Harvard Medical School, Boston

Kenneth H. Mayer, M.D.

Brigham and Women's Hospital, Harvard University Center for AIDS Research, Harvard Medical School, Harvard School of Public Health, and Fenway Health, Boston

Copyright $\odot 2013$ Massachusetts Medical Society

Address reprint requests to Dr. Walensky at Massachusetts General Hospital, Medical Practice Evaluation Center, Division of Infectious Disease, 50 Staniford St., 9th Flr., Boston, MA 02114, or at rwalensky @ partners.org..

Disclosure forms provided by the authors are available with the full text of this article at NEJM.org. 
Ying Q. Chen, Ph.D.

Department of Medicine, Faculty of Health Sciences, University of the Witwatersrand, Johannesburg; Vaccine and Infectious Disease Division, Fred Hutchinson Cancer Research Center, Seattle

Lei Wang, Ph.D.

Department of Medicine, Faculty of Health Sciences, University of the Witwatersrand, Johannesburg; Vaccine and Infectious Disease Division, Fred Hutchinson Cancer Research Center, Seattle

Marybeth McCauley, M.P.H.

FHI 360, Washington, DC

Theresa Gamble, Ph.D.

FHI 360, Durham, NC

George R. Seage III, D.Sc., M.P.H.

Department of Epidemiology, Center for Biostatistics in AIDS Research, Boston

Myron S. Cohen, M.D.

University of North Carolina School of Medicine, Chapel Hill

Kenneth A. Freedberg, M.D.

Divisions of Infectious Disease, General Medicine, Medical Practice Evaluation Center, Brigham and Women's Hospital, Harvard University Center for AIDS Research, Harvard Medical School, Departments of Health Policy and Management, Boston

\section{Abstract}

BACKGROUND-The cost-effectiveness of early antiretroviral therapy (ART) in persons infected with human immunodeficiency virus (HIV) in serodiscordant couples is not known. Using a computer simulation of the progression of HIV infection and data from the HIV Prevention Trials Network 052 study, we projected the cost-effectiveness of early ART for such persons.

METHODS-For HIV-infected partners in serodiscordant couples in South Africa and India, we compared the early initiation of ART with delayed ART. Five-year and lifetime outcomes included cumulative HIV transmissions, life-years, costs, and cost-effectiveness. We classified early ART as very cost-effective if its incremental cost-effectiveness ratio was less than the annual per capita gross domestic product (GDP; $\$ 8,100$ in South Africa and $\$ 1,500$ in India), as costeffective if the ratio was less than three times the GDP, and as cost-saving if it resulted in a decrease in total costs and an increase in life-years, as compared with delayed ART.

RESULTS-In South Africa, early ART prevented opportunistic diseases and was cost-saving over a 5-year period; over a lifetime, it was very cost-effective ( $\$ 590$ per life-year saved). In India, early ART was cost-effective ( $\$ 1,800$ per life-year saved) over a 5-year period and very costeffective ( $\$ 530$ per life-year saved) over a lifetime. In both countries, early ART prevented HIV transmission over short periods, but longer survival attenuated this effect; the main driver of lifeyears saved was a clinical benefit for treated patients. Early ART remained very cost-effective over a lifetime under most modeled assumptions in the two countries.

CONCLUSIONS-In South Africa, early ART was cost-saving over a 5-year period. In both South Africa and India, early ART was projected to be very cost-effective over a lifetime. With individual, public health, and economic benefits, there is a compelling case for early ART for serodiscordant couples in resource-limited settings. (Funded by the National Institute of Allergy and Infectious Diseases and others.) 
IN THE HUMAN IMMUNODEFICIENCY VIRUS (HIV) Prevention Trials Network (HPTN) 052 study, ${ }^{1}$ early antiretroviral therapy (ART), as compared with delayed ART, was associated with a $96 \%$ relative reduction in the rate of linked transmission among serodiscordant couples during 24 months of follow-up. (Early ART was defined as therapy initiated when the CD4+ T-cell count ranged from 350 to 550 per cubic millimeter, and delayed ART as therapy initiated when the CD4+ count was <250 per cubic millimeter.) The trial also showed clear clinical benefits for patients with HIV infection in the early-ART group, as compared with those in the delayed-ART group, since the early initiation of therapy prevented both primary and secondary complications of HIV infection. ${ }^{2}$ Results from observational studies have also supported the efficacy of early HIV treatment. ${ }^{3-6}$

Although the clinical efficacy of treatment as prevention may be consistent across settings, the economic value may differ, owing to differences in resource utilization, labor costs, and ability to pay (as it relates to different values of the national gross domestic product [GDP]). We projected the cost-effectiveness of early ART, as compared with delayed ART, among serodiscordant couples, accounting for both treatment and transmission effects in South Africa and India, two of the nine countries that were included in the HPTN 052 study.

\section{METHODS}

\section{ANALYTIC OVERVIEW}

In collaboration with the HPTN 052 investigators, the Cost-Effectiveness of Preventing AIDS Complications (CEPAC) International Group conducted a model-based analysis that closely mirrored the HPTN 052 trial. We used a microsimulation model of HIV disease, treatment, and transmission ${ }^{7-10}$ to compare early ART with delayed ART in serodiscordant couples. We conducted analyses to determine whether regional differences in South Africa and India had an effect on the value of early ART versus delayed ART. We evaluated the following outcomes from a modified societal perspective (excluding time and productivity costs): clinical outcomes (the rate of survival and per-person life expectancy in life-years), transmission outcomes (first- and second-order transmission events), and economic outcome (per-person HIV-related health care costs). We first simulated the trial period (24 months of follow-up) and then projected these outcomes over a 5-year period and over a lifetime (i.e., until every member of the simulated index cohort died).

We calculated the incremental cost-effectiveness ratio of early ART, as compared with delayed ART, as the change in cost divided by the change in life-years, accounting for both the health gains and medical costs for the treated patient and for the timing, decreased survival, and increased costs of the projected transmission events (see the Supplementary Appendix, available with the full text of this article at NEJM.org). We classified early ART as very cost-effective if the incremental cost-effectiveness ratio was less than the per capita GDP in 2011 (\$8,100 for South Africa and \$1,500 for India) and as cost-effective if the incremental cost-effectiveness ratio was less than three times the GDP. ${ }^{11-13}$ Early ART was considered to be cost-saving, as compared with delayed ART, when it led to both a decrease in total costs and an increase in life-years. For cost-effectiveness calculations, costs and life expectancy of both index and transmitted cases were discounted (i.e., given less value in the future) at an annual rate of $3 \%$; survival) were not discounted.

\section{CEPAC INTERNATIONAL MODEL}

Disease Model-The CEPAC International model is a microsimulation of HIV disease progression and treatment that is focused on resource-limited settings. ${ }^{7-10}$ Simulated patients are generated with random draws of characteristics from distributions of age, sex, CD4+ 
count, and HIV RNA level (see the Supplementary Appendix). The HIV RNA level determines the monthly change in the CD4+ count. A lower count leads to a higher risk of setting-specific opportunistic diseases and HIV-related mortality. The model simulates a patient's underlying CD4+ count and HIV RNA level; however, clinical decisions are based on presentation with an opportunistic disease or on an observed CD4+ count and HIV RNA level, which are measured on periodic laboratory testing (see the Supplementary Appendix).

According to the HPTN 052 study protocol, CD4+ counts and HIV RNA levels in the model were monitored quarterly; when a patient met strategy-specific treatment criteria (e.g., a CD4+ count of $<250$ per cubic millimeter in the delayed-ART group), ART was initiated. Once patients were receiving ART, they had an initial probability of virologic suppression, with a corresponding increase in CD4+ counts. Among these patients, there was a small monthly probability of loss of treatment efficacy (late failure), resulting in virologic rebound and subsequent decline in the CD4+ count. At the time of laboratory-confirmed treatment failure, as defined in the trial, ${ }^{1}$ patients were started on a second-line protease inhibitorbased drug regimen with an efficacy that was assumed to be equivalent to that of the firstline therapy (see the Supplementary Appendix). Patients could be categorized as having been lost to follow-up before starting ART or while receiving therapy; in the latter group, therapy was discontinued. Patients who were lost to follow-up had a 50\% probability of returning to care during the month of an acute episode of an opportunistic disease.

Transmission Module-We used a transmission module to tally monthly transmission events to primary and secondary partners and to project both the incident transmission from the index patient (i.e., first-order transmission) and subsequent transmissions to other uninfected partners (second-order transmission) (for details, see the Supplementary Appendix). The module allowed for variation in the duration of primary partnerships and in sexual activity outside those partnerships, age-adjusted sexual behaviors, and transmissibility. For incident HIV cases, acute infection increased infectivity to future uninfected partners (see the Supplementary Appendix). On resolution of acute disease, previously uninfected incident cases - once HIV was detected - were eligible for the same strategy (early vs. delayed ART) as the index case. In the case of secondary partners, we assumed that early ART would benefit all partners of the HIV-infected person.

\section{INPUT DATA}

Cohort-Characteristics of the study cohort reflected the characteristics of all participants in the HPTN 052 study. For patients in both South Africa and India, we used pooled estimates of mean $( \pm \mathrm{SD})$ age $(33.7 \pm 8.5$ years) and initial CD4+ count $(449 \pm 120$ per cubic millimeter). Because there were significant differences between the two countries with respect to patients' sex and HIV RNA distributions, we used country-specific data for these variables (Table 1). ${ }^{14-21}$

Natural History-We derived monthly rates of opportunistic diseases from the widely reported Cape Town AIDS Cohort in South Africa and the Y.R. Gaitonde Centre for AIDS Research and Education (YRG CARE) cohort in India. ${ }^{22,23}$ For patients who were receiving treatment, the use of trimethoprim-sulfamethoxazole (which was initiated at a CD4+ count of $<200$ per cubic millimeter) ${ }^{1,14,24,25}$ attenuated the frequency of some events. ${ }^{26}$

ART Efficacy and Loss to Follow-up-We derived data on the efficacy of ART and loss to follow-up from the trial (see the Supplementary Appendix). At 48 weeks, the rate of virologic suppression was $92 \%$. The rate of later virologic failure was 1.4 cases per 100 person-years. The rate of loss to follow-up was 3.4 cases per 100 person-years. 
Transmission-Data from the HPTN 052 study showed that the average number of secondary partners per month was 0.014 in South Africa and 0.007 in India. Within a serodiscordant partnership, trial-derived transmission rates depended on the HIV RNA level of the index patient, with rates ranging from 0.10 cases per 100 person-years for patients with virologic suppression (<400 copies per milliliter) to 1.48 per 100 person-years for patients without virologic suppression. We assumed that sexual activity was agedependent ${ }^{18}$ and that the average duration of the primary partnership was 15 years. ${ }^{15} \mathrm{We}$ also assumed that for the incident (first-order) cases that were not in primary partnerships, the average number of partners per month was $2.0 .{ }^{27}$ We modeled a 5-month duration of acute infection, during which infectivity increased by a factor of 26 , as compared with infectivity among patients without HIV RNA suppression. ${ }^{16,17}$

Costs-We calculated the costs of treating a patient with HIV infection by multiplying resources used (e.g., inpatient days, outpatient visits, and laboratory tests) by countryspecific unit costs that included costs for professional time, supplies, and non-ART medications, as well as capital costs; these values were derived from data obtained from sources other than the HPTN 052 study. ${ }^{19,20}$ We derived ART costs from the World Health Organization (WHO) Global Price Reporting Mechanism database (Table 1). ${ }^{21}$ We used country-specific GDP deflators and the average 2011 exchange rate to convert all currencies to 2011 U.S. dollars. ${ }^{11,28}$

Sensitivity and Scenario Analyses-We first conducted an analysis for the base-case scenario, as defined by the input values described in Table 1. We then conducted one-way sensitivity analyses of clinical, transmission-related, and cost variables to examine their effect on four outcomes of delayed ART, as compared with early ART: the 5-year and lifetime change in cumulative transmissions and the 5-year and lifetime incremental costeffectiveness ratio.

Since clinical trials probably represent the best-case scenario with regard to ART efficacy, transmissions, and other outcomes, we examined two additional scenarios (multiway sensitivity analyses) (Table S2 in the Supplementary Appendix). In the routine-care scenario, we used a later presentation for care (mean CD4+ count per cubic millimeter, 195 in South Africa and 305 in India). ${ }^{23,29}$ For the two countries, we used a 48-week virologic suppression rate of $74 \%$, a later virologic failure rate of 9.5 cases per 100 person-years, ${ }^{30} \mathrm{a}$ rate for loss to follow-up of 10.0 cases per 100 person-years through the first year of ART (and 3.2 per 100 person-years thereafter), ${ }^{31-33}$ and a slightly higher transmission rate (0.16 to 9.03 cases per 100 person-years). ${ }^{34}$ In the worst-case scenario, we incorporated cohort characteristics that were similar to those in the routine-care scenario: a 48 -week virologic suppression rate of $65 \%$, a later failure rate of 14.1 cases per 100 person-years, ${ }^{35}$ a rate of loss to follow-up of 10.0 cases per 100 person-years in the first year of ART (and 5.1 per 100 person-years thereafter), ${ }^{31-33}$ and a transmission rate of 1.25 to 21.83 cases per 100 person-years. ${ }^{18}$

\section{RESULTS \\ SIMULATION OF TRIAL PERIOD}

On the basis of clinical and ART data from the HPTN 052 study, the model produced a plausible replication of trial-based, 24-month immunologic outcomes (Fig. S1 in the Supplementary Appendix). 


\section{PROJECTED OUTCOMES FOR INDEX PATIENTS}

Clinical Factors-In South Africa, the 5-year rate of survival among index patients increased with early ART, as compared with delayed ART (93\% vs. $83 \%$ ). Over a lifetime, the life expectancies with early ART, as compared with delayed ART, were 189.4 months and 165.2 months, respectively. Clinical outcomes in India were qualitatively similar to those in South Africa (Table 2).

Costs-In South Africa, early ART prevented many opportunistic diseases (e.g., tuberculosis), thus preventing comparatively high costs of care (Fig. S2 in the Supplementary Appendix), a savings that more than outweighed ART costs. Consequently, over a 5-year period, early ART and delayed ART in South Africa had similar per-person costs (approximately $\$ 4,800$ ). Over a lifetime, the longer survival that was associated with early ART increased total costs, as compared with delayed ART ( $\$ 16,600$ vs. $\$ 15,100)$ (Table 2).

In India, the average costs associated with medical care for opportunistic diseases, as compared with costs associated with ART, are lower than those in South Africa (Fig. S2 in the Supplementary Appendix). Although early ART prevented opportunistic diseases in India, the cost savings did not outweigh the ART costs; thus, early ART led to higher total costs than delayed ART over both a 5-year period $(\$ 2,200$ vs. $\$ 1,800)$ and a lifetime $(\$ 8,400$ vs. $\$ 7,300)$ (Table 2).

Transmissions-In South Africa, early ART resulted in an immediate, large decrease in the number of projected transmissions (Fig. 1A, and Fig. S4A in the Supplementary Appendix). The difference in transmission rates was attenuated over time, as more patients started receiving therapy in the delayed-ART group. By year 6 after presentation, rates of annual transmissions were greater in the early-ART group than in the delayed-ART group because most patients in the delayed-ART group were receiving ART and some patients in the early-ART group had begun to have virologic failure. However, rates of cumulative transmissions in the early-ART group remained consistently lower than those in the delayedART group throughout the lifetime of the index patient (Fig. 1B). Projected transmission results were similar in the two countries (Fig. S3A, S3B, and S4B in the Supplementary Appendix).

Cost-Effectiveness-When we included transmission-related life-months lost and costs accrued over a 5-year period, early ART was cost-saving, as compared with delayed ART, in South Africa. Over a lifetime, early ART was very cost-effective (\$590 per life-year saved) (Table 2, and Fig. S6 in the Supplementary Appendix). In India, early ART was costeffective over a 5-year period ( $\$ 1,800$ per life-year saved) and very cost-effective over a lifetime (\$530 per life-year saved) (Table 2, and Fig. S6 in the Supplementary Appendix).

In the two countries and over the two time periods, the clinical benefit to the index patient who received early ART was much larger in magnitude than the clinical benefit associated with the prevention of new transmissions. For example, for a patient receiving early ART in South Africa, the 5-year increase in life expectancy of 3.2 months (from 51.7 to 54.9 months) was much larger than the clinical benefit associated with the prevention of new transmissions, which resulted in 0.07 life-months gained (a decrease in life-months lost from 0.09 to 0.02 ).

\section{SENSITIVITY ANALYSES}

Transmissions-In the South African base-case scenario, early ART, as compared with delayed ART, resulted in a relative reduction of $69 \%$ in the number of transmissions over a 
5-year period and of $13 \%$ over a lifetime (Table 2). Input values related to treatment specifically, changes in 48-week suppression rates, late virologic failure (after initial suppression), and loss to follow-up - had the greatest effect on these results (Fig. S7A and S7B in the Supplementary Appendix). Regardless of these variations, early ART consistently resulted in fewer infections than delayed ART over the 5-year and lifetime periods. Similar results were seen in India (Fig. S7C and S7D in the Supplementary Appendix).

Cost-Effectiveness-In South Africa, in most of the ranges that we examined, early ART was cost-saving over a 5-year period. Variation in several treatment-related variables (including increased rates of late failure associated with poor ART adherence) moved the incremental cost-effectiveness ratio for early ART from cost-saving to very cost-effective; however, for transmission-related variables, these ratios were consistently cost-saving (Fig. $2 \mathrm{~A})$. Five-year results were also sensitive to these variables in India, where some variation in treatment-related variables moved early ART from cost-effective to very cost-effective (Fig. 2B). In one-way sensitivity analyses, early ART was very cost-effective over a lifetime across all ranges of variables that were examined in the two countries (Fig. S8A and S8B in the Supplementary Appendix).

\section{SCENARIO ANALYSES}

In the two countries and for both time periods, projected clinical outcomes were worse for alternative scenarios in which there were lower rates of efficacy for ART, higher rates of late virologic failure, and higher rates of loss to follow-up (see Table S3 for the routine-care scenario and Table S4 for the worst-case scenario in the Supplementary Appendix). With later ART initiation, less effective treatment, and higher transmission levels, early ART had more modest benefits with respect to transmission at 5 years, with no benefit over a lifetime (Fig. S5 in the Supplementary Appendix). Despite these changes in variables and subtle differences in outcome, economic and policy conclusions were robust. In the base-case scenario, as well as in the routine-care and worst-case scenarios, early ART at 5 years was cost-saving in South Africa and cost-effective in India. In all scenarios and in both countries, early ART was very cost-effective over a lifetime (Tables S3 and S4 in the Supplementary Appendix).

\section{DISCUSSION}

With compelling evidence supporting the clinical and preventive benefits of early ART, the focus is now on economic questions with respect to early ART. ${ }^{3-6}$ In a model-based analysis projecting the clinical, population, and economic effects of the HPTN 052 study in South Africa and India, we found that early ART substantially improved the rate of survival of infected patients, greatly decreased the rate of early HIV transmissions, and provided an excellent return on investment. We specifically chose to model these two countries because they had the highest numbers of persons with HIV infection among the nine countries that were evaluated in the HPTN 052 study and because South Africa and India were representative of middle-income and lower-income countries, respectively (Table S5 in the Supplementary Appendix). ${ }^{11}$ Over the first 5 years, the differences in costs of care and GDP accounted for differences in the cost-effectiveness of early ART. In South Africa, early ART was found to be cost-saving, whereas in India, early ART was cost-effective. Over the long term, early ART was very cost-effective in both countries.

Data from the HPTN 052 study highlight the benefits of early ART with respect to both morbidity and transmission. Early ART significantly increased the time to a diagnosis of tuberculosis, a WHO stage 4 event, or secondary complications, as compared with delayed 
ART. ${ }^{2}$ In our model-based, longer-term projections, these delays in morbidity led to substantial reductions in mortality - differences that were not detected in the short timeframe of the HPTN 052 study. Indeed, these data and projections underscore that among the greatest prevention effects of early ART is the durable prevention of opportunistic diseases.

Our analyses show that the preventive value of early ART depends on the extent to which patients remain in care and receive effective treatment. The capacity to maintain virologic suppression among patients who are retained in care will determine the magnitude of longterm benefits with respect to transmission.

Although our study shows that early ART is very cost-effective, a major challenge to its implementation - in addition to the challenge of earlier HIV disease detection ${ }^{36}$ - is affordability in resource-limited settings. This should not be the case in South Africa. Over the first 5 years, the cost of early ART is more than offset by the prevention of common, expensive-to-treat opportunistic diseases; the opportunity to offset those costs is lost when ART is delayed. In India, upfront expenditures will be needed to implement early ART.

Our results are more conservative - with respect to cumulative transmissions prevented by early ART — than those modeled in studies reported previously. ${ }^{37,38}$ The reason for this is that we accounted for the individual benefit of early ART and the monthly changes in virologic-based transmissions associated with HIV detection, virologic suppression and rebound, and loss to follow-up. We also specifically chose to examine countries with different care and cost structures in order to show the generalizability of these results in two very different resource-limited settings.

In the HPTN 052 study, a total of 39 HIV transmission events were reported, with definitive HIV phylogenetic data available for 36 of these events. Of the latter transmission events, 7 $(19 \%)$ were not phylogenetically linked to the primary partner and were presumed to have been acquired from secondary partners, ${ }^{39}$ whose characteristics are unknown. If many of these secondary partners would not be eligible for ART under a policy of early ART for serodiscordant couples, then we may have overestimated the preventive effect of this policy. Alternatively, if the secondary partners would be eligible for ART as members of serodiscordant couples, then we may have underestimated the preventive effect of early ART. Regardless of the direction of this bias, our results and sensitivity analyses showed that early ART would be very cost-effective.

Our study has several limitations. First, we intentionally adhered closely to the design, population, and data of the HPTN 052 study. ${ }^{1}$ Although we have examined the potential generalizability of the conclusions in routine-care and worst-case scenarios, we cannot be sure that the results are representative of care in nontrial settings or of patients with HIV who are not in regular serodiscordant partnerships. Second, in the scenario analyses, we did not consider the possibility of increased drug resistance because of poor adherence. Third, since HIV-infected patients were already identified in the HPTN 052 study, we consider our study to be an analysis of "treatment as prevention" rather than an analysis of "test and treat." Fourth, although we excluded productivity and other nonmedical economic benefits of prevented transmissions, the inclusion of such factors would make the results even more economically attractive..$^{40}$ Fifth, despite the many benefits of modeling HIV progression together with transmissions, such a complex model is not easily conducive to probabilistic sensitivity analysis. Finally, we limited the analysis to first-order and second-order transmissions from the index patient; as indicated by the modest effect of second-order transmissions, the inclusion of more distal transmissions would no doubt have a minimal effect on the results. 
In conclusion, our analysis using the clinical data from the HPTN 052 study and published data from cohorts in South Africa and India shows that early ART provides substantial individual and population benefits over delayed ART. The ultimate preventive value of early ART is a shared responsibility of providers and patients, and patients who remain in care with virologic suppression will glean the maximum long-term preventive effect of early ART. Early ART also has major economic benefits and is very cost-effective - results that are robust across settings and assumptions.

\section{Supplementary Material}

Refer to Web version on PubMed Central for supplementary material.

\section{Acknowledgments}

National Institute of Allergy and Infectious Diseases (NIAID)

R01 AI058736, R01 AI093269, and UM1 AI068636

National Institute of Mental Health

R01 MH087328

(all to the CEPAC International Group), by the HIV Prevention Trials Network (HPTN), and by grants from NIAID

UM1-AI068619, U01-AI068619, UM1-AI068613, and U01-AI068613

HPTN Network Laboratory;

UM1-AI068617 and U01-AI068617

HPTN Statistical and Data Management Center) and by a grant from the AIDS Clinical Trials Group

UM1 AI068636

Study drugs for the HPTN 052 trial were donated by Abbott Laboratories, Boehringer Ingelheim, Bristol-Myers Squibb, Gilead Sciences, GlaxoSmithKline/ViiV Healthcare, and Merck.

We thank the HPTN 052 study team and the study participants.

\section{REFERENCES}

1. Cohen MS, Chen YQ, McCauley M, et al. Prevention of HIV-1 infection with early antiretroviral therapy. N Engl J Med. 2011; 365:493-505. [PubMed: 21767103]

2. Grinsztejn, B.; Hosseinipour, M.; Swindells, S., et al. Proceedings of the XIX International AIDS Conference. Washington, DC: Jul 21-27. 2012 Effect of early versus delayed initiation of antiretroviral therapy (ART) on clinical outcomes in the HPTN 052 randomized clinical trial. abstract

3. Cain LE, Logan R, Robins JM, et al. When to initiate combined antiretroviral therapy to reduce mortality and AIDS-defining illness in HIV-infected persons in developed countries: an observational study. Ann Intern Med. 2011; 154:509-15. [PubMed: 21502648]

4. Kitahata MM, Gange SJ, Abraham AG, et al. Effect of early versus deferred antiretroviral therapy for HIV on survival. N Engl J Med. 2009; 360:1815-26. [PubMed: 19339714]

5. Writing Committee for the CASCADE Collaboration. Timing of HAART initiation and clinical outcomes in human immunodeficiency virus type 1 seroconverters. Arch Intern Med. 2011; 171:1560-9. [PubMed: 21949165]

6. Donnell D, Baeten JM, Kiarie J, et al. Heterosexual HIV-1 transmission after initiation of antiretroviral therapy: a prospective cohort analysis. Lancet. 2010; 375:2092-8. [PubMed: 20537376] 
7. Ademi Z, Kim H, Zomer E, Reid CM, Hollingsworth B, Liew D. Overview of pharmacoeconomic modelling methods. Br J Clin Pharmacol. 2013; 75:944-50. [PubMed: 22882459]

8. Goldie SJ, Yazdanpanah Y, Losina E, et al. Cost-effectiveness of HIV treatment in resource-poor settings — the case of Côte d'Ivoire. N Engl J Med. 2006; 355:1141-53. [PubMed: 16971720]

9. Walensky RP, Wood R, Weinstein MC, et al. Scaling up antiretroviral therapy in South Africa: the impact of speed on survival. J Infect Dis. 2008; 197:1324-32. [PubMed: 18422445]

10. Walensky RP, Wood R, Ciaranello AL, et al. Scaling up the 2010 World Health Organization HIV Treatment Guidelines in resource-limited settings: a model-based analysis. PLoS Med. 2010; 7(12):e1000382. [PubMed: 21209794]

11. International Monetary Fund. World Economic Outlook Database. 2012. (http://www.imf.org/ external/pubs/ft/weo/2012/02/weo da ta/index. aspx).

12. Gold, MR.; Siegel, J.; Russell, L.; Weinstein, M. Cost effectiveness in health and medicine. Oxford University Press; New York: 1996.

13. Commission on Macroeconomics and Health. Macroeconomics and health: investing in health for economic development. World Health Organization; Geneva: 2001.

14. Preventing sexual transmission of HIV with anti-HIV drugs. 2012. ClinicalTrials.gov(http:// clinicaltrials.gov/show/NCT00074581).

15. Lurie MN, Williams BG, Zuma K, et al. Who infects whom? HIV-1 concordance and discordance among migrant and non-migrant couples in South Africa. AIDS. 2003; 17:2245-52. [PubMed: 14523282]

16. Powers KA, Ghani AC, Miller WC, et al. The role of acute and early HIV infection in the spread of HIV and implications for transmission prevention strategies in Lilongwe, Malawi: a modelling study. Lancet. 2011; 378:256-68. [PubMed: 21684591]

17. Hollingsworth TD, Anderson RM, Fraser C. HIV-1 transmission, by stage of infection. J Infect Dis. 2008; 198:687-93. [PubMed: 18662132]

18. Gray RH, Wawer MJ, Brookmeyer R, et al. Probability of HIV-1 transmission per coital act in monogamous, heterosexual, HIV-1-discordant couples in Rakai, Uganda. Lancet. 2001; 357:114953. [PubMed: 11323041]

19. Medicins Sans Frontieres and Health Systems Trust. Cost-effectiveness of antiretroviral treatment for HIV-positive adults in a South African township. 2004. (http://www.healthlink.org.za/uploads/ files/arv_cost.pdf).

20. YRG CARE treatment costs 2009-2012. YRG CARE; Chennai, India: 2012.

21. World Health Organization. Global price reporting mechanism for HIV, tuberculosis, and malaria. (http://www.who.int/hiv/amds/gprm/en/)

22. Holmes CB, Wood R, Badri M, et al. CD4 decline and incidence of opportunistic infections in Cape Town, South Africa: implications for prophylaxis and treatment. J Acquir Immune Defic Syndr. 2006; 42:464-9. [PubMed: 16810113]

23. Cecelia AJ, Christybai P, Anand S, et al. Usefulness of an observational database to assess antiretroviral treatment trends in India. Natl Med J India. 2006; 19:14-7. [PubMed: 16570679]

24. National AIDS Control Organisation. Guidelines for prevention and management of common opportunistic infections/malignancies among HIV-infected adult and adolescent. Ministry of Health \& Family Welfare; New Delhi, India: May. 2007

25. Clinical guidelines for the management of HIV \& AIDS in adults and adolescents. National Department of Health; Pretoria, South Africa: 2010. (http://www.who.int/hiv/pub/guidelines/ south_africa_art.pdf)

26. Yazdanpanah Y, Losina E, Anglaret X, et al. Clinical impact and cost-effectiveness of cotrimoxazole prophylaxis in patients with HIV/AIDS in Côte d'Ivoire: a trial-based analysis. AIDS. 2005; 19:1299-308. [PubMed: 16052085]

27. Chopra M, Townsend L, Johnston L, et al. Estimating HIV prevalence and risk behaviors among high-risk heterosexual men with multiple sex partners: use of respondent-driven sampling. J Acquir Immune Defic Syndr. 2009; 51:72-7. [PubMed: 19282783]

28. OANDA. Historical exchange rates. (http://www.oanda.com/currency/historical-rates) 
29. Fairall LR, Bachmann MO, Louwagie GM, et al. Effectiveness of antiretroviral treatment in a South African program: a cohort study. Arch Intern Med. 2008; 168:86-93. [PubMed: 18195200]

30. Barth RE, van der Loeff MF, Schuur-man R, Hoepelman AI, Wensing AM. Virological follow-up of adult patients in antiretroviral treatment programmes in sub-Saharan Africa: a systematic review. Lancet Infect Dis. 2010; 10:155-66. [PubMed: 20185094]

31. Rosen S, Fox MP, Gill CJ. Patient retention in antiretroviral therapy programs in sub-Saharan Africa: a systematic review. PLoS Med. 2007; 4(10):e298. [PubMed: 17941716]

32. Brinkhof MW, Pujades-Rodriguez M, Egger M. Mortality of patients lost to follow-up in antiretroviral treatment programmes in resource-limited settings: systematic review and metaanalysis. PLoS One. 2009; 4(6):e5790. [PubMed: 19495419]

33. Fox MP, Rosen S. Patient retention in antiretroviral therapy programs up to three years on treatment in sub-Saharan Africa, 2007-2009: systematic review. Trop Med Int Health. 2010; 15(Suppl 1):1-15. [PubMed: 20586956]

34. Attia S, Egger M, Müller M, Zwahlen M, Low N. Sexual transmission of HIV according to viral load and antiretroviral therapy: systematic review and meta-analysis. AIDS. 2009; 23:1397-404. [PubMed: 19381076]

35. Nachega JB, Hislop M, Nguyen H, et al. Antiretroviral therapy adherence, virologic and immunologic outcomes in adolescents compared with adults in southern Africa. J Acquir Immune Defic Syndr. 2009; 51:65-71. [PubMed: 19282780]

36. Mugglin, C.; Althoff, K.; Wools-Kaloustian, K., et al. Immunodeficiency at the start of ART: global view; Program and abstracts of the 19th Conference on Retroviruses and Opportunistic Infections, Seattle; Mar 5-8. 2012 abstract

37. Granich RM, Gilks CF, Dye C, De Cock KM, Williams BG. Universal voluntary HIV testing with immediate antiretroviral therapy as a strategy for elimination of HIV transmission: a mathematical model. Lancet. 2009; 373:48-57. [PubMed: 19038438]

38. Schwartländer B, Stover J, Hallett T, et al. Towards an improved investment approach for an effective response to HIV/AIDS. Lancet. 2011; 377:2031-41. [PubMed: 21641026]

39. Eshleman SH, Hudelson SE, Redd AD, et al. Analysis of genetic linkage of HIV from couples enrolled in the HIV Prevention Trials Network 052 trial. J Infect Dis. 2011; 204:1918-26. [PubMed: 21990420]

40. Thirumurthy H, Galárraga O, Larson B, Rosen S. HIV treatment produces economic returns through increased work and education, and warrants continued US support. Health Aff (Millwood). 2012; 31:1470-7. [PubMed: 22778336] 


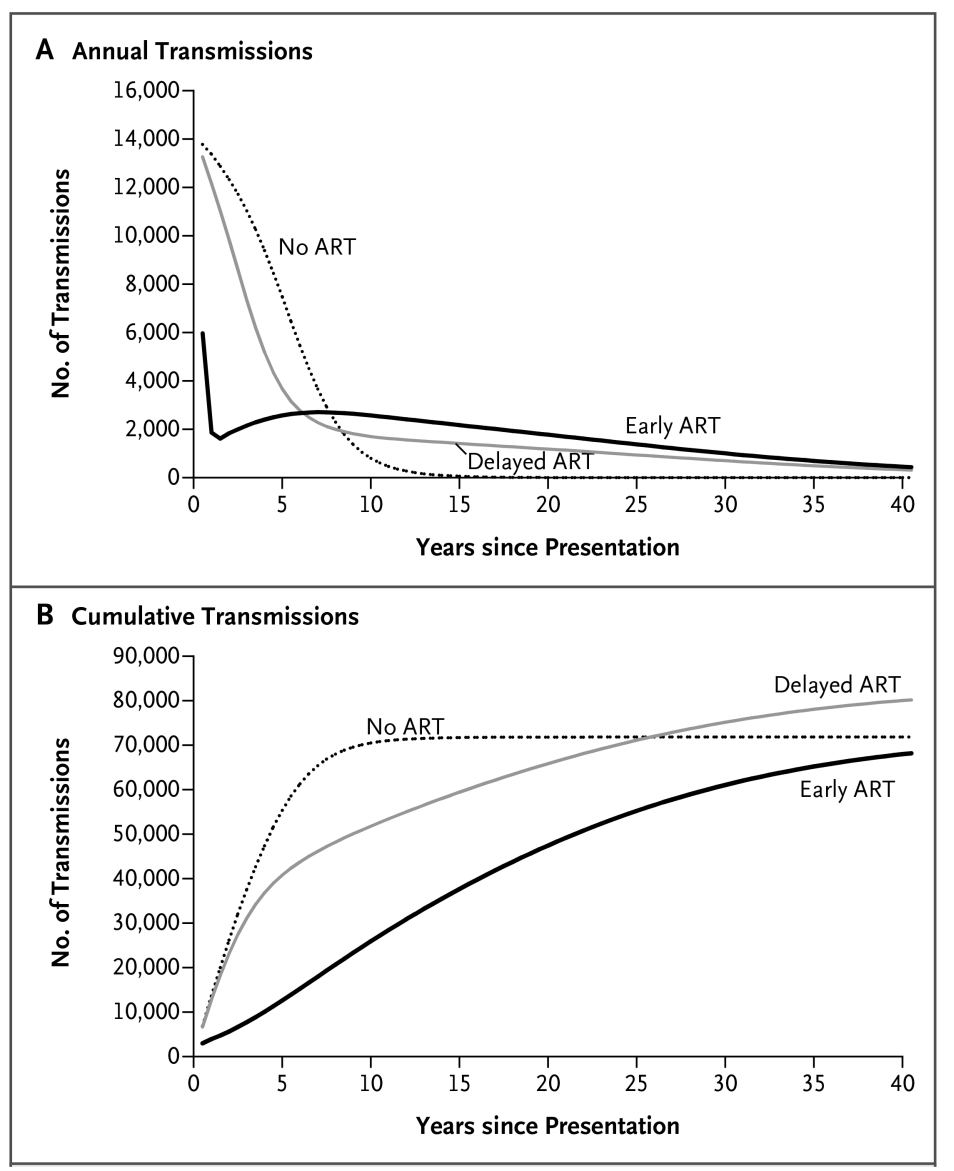

Figure 1. HIV Transmissions among Serodiscordant Couples in South Africa, According to the Strategy for the Initiation of Antiretroviral Therapy (ART).

Shown are results for a modeled cohort of 1 million HIV-infected index patients in serodiscordant relationships, according to whether they received no ART, delayed ART, or early ART. Panel A shows the total number of transmissions per year, and Panel B shows the cumulative number of transmissions. The number of transmissions includes both firstorder and second-order transmissions. 


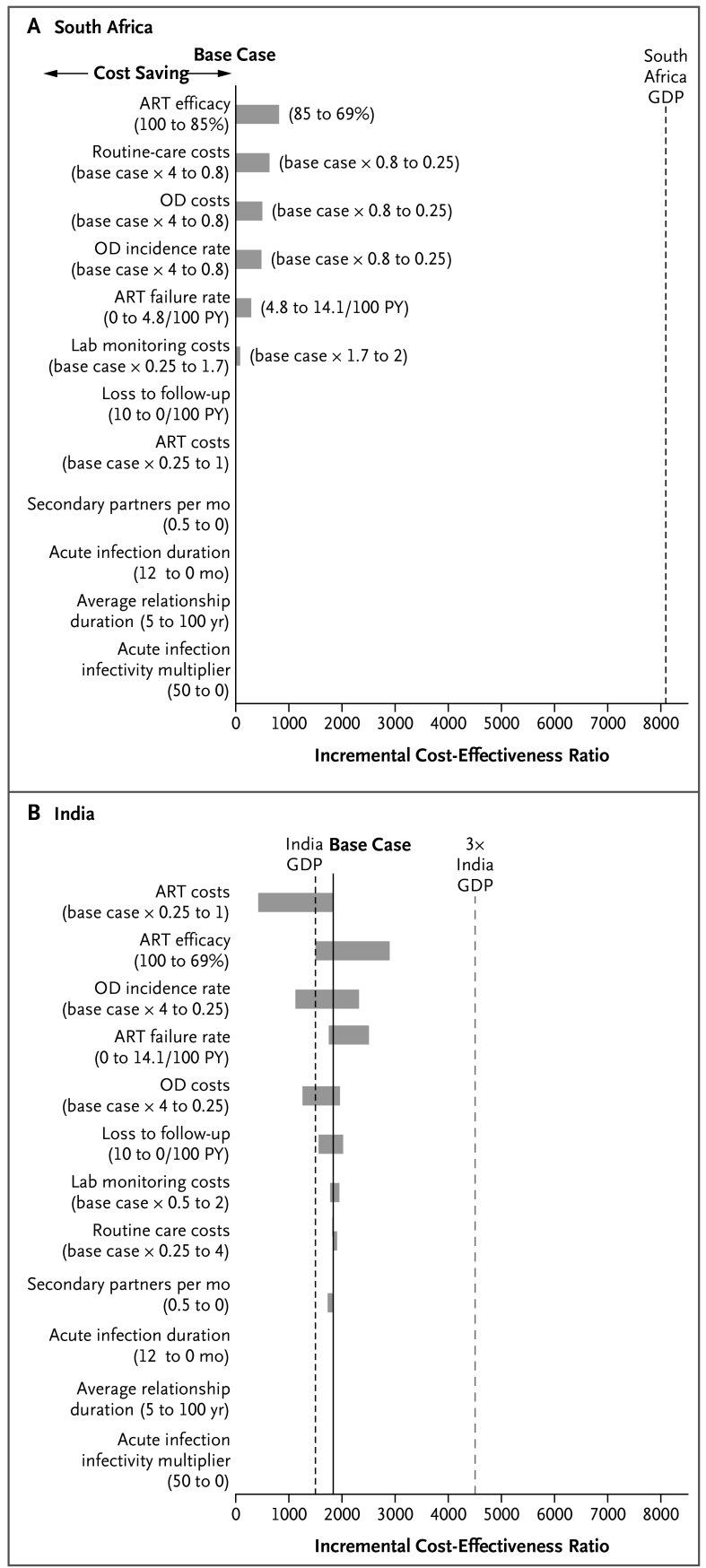

Figure 2. Results of One-Way Sensitivity Analyses.

Shown are the effects of various treatment and transmission variables on the incremental cost-effectiveness ratio (the change in cost divided by the change in life-years) for early ART, as compared with delayed ART, over a 5-year period in South Africa (Panel A) and India (Panel B). ART efficacy was defined as HIV RNA suppression at 48 weeks. In these tornado diagrams (so named because the data are listed vertically in a funnel pattern, with the most important variables at the top), the solid vertical line indicates the incremental costeffectiveness ratio for the base-case scenario in the study. The length of the horizontal bars indicates the range of incremental cost-effectiveness ratios over the specified range of variable values; the longer the bar, the greater the sensitivity of the incremental cost- 
effectiveness ratio to that variable. To the right of the horizontal bars in Panel A, the range of values that were examined is shown in parentheses, with the value producing the lower incremental cost-effectiveness ratio listed first. Incremental cost-effectiveness ratios that met the definition of cost-saving (which was defined as both a decrease in total costs and an increase in life-years) are not shown in the tornado diagram. Instead, the cost-saving range for incremental cost-effectiveness ratios is provided to the left of the horizontal bars. The dashed vertical black line indicates the gross domestic product (GDP) of the country (the threshold for being very cost-effective), and the dashed vertical red line represents three times the GDP of the country (the threshold for being cost-effective, which is shown for India only). The GDPs are shown in dollars (see the Methods section). In South Africa, numerous ranges of values that were examined, including all transmission variables, showed that early ART was cost-saving over a 5-year period. In India, early ART, as compared with delayed ART, was found to be cost-effective over a 5-year period for the base case; with variation in some treatment variables, early ART was very cost-effective $(<\$ 1,500$ per lifeyear saved). OD denotes opportunistic disease, and PY person-year. 
Table 1

Base-Case Input Data for Analysis of the Cost-Effectiveness of Early ART in the HPTN 052 Trial. ${ }^{*}$

\begin{tabular}{|c|c|c|c|}
\hline \multirow[t]{2}{*}{ Variable } & \multicolumn{2}{|c|}{ Base-Case Value ${ }^{\dagger}$} & \multirow[t]{2}{*}{ Range } \\
\hline & South Africa & India & \\
\hline \multicolumn{4}{|l|}{ Cohort characteristic } \\
\hline Age (yr) & $33.7 \pm 8.5$ & $33.7 \pm 8.5$ & \\
\hline Initial CD4+ count $\left(\right.$ cells $\left./ \mathrm{mm}^{3}\right)$ & $449 \pm 120$ & $449 \pm 120$ & \\
\hline \multicolumn{4}{|l|}{$\operatorname{Sex}(\%)$} \\
\hline Male & 42 & 67 & \\
\hline Female & 58 & 33 & \\
\hline \multicolumn{4}{|l|}{ Efficacy of first- and second-line ART } \\
\hline Patients with HIV RNA suppression at $48 \mathrm{wk}(\%)$ & 92 & 92 & $69-100$ \\
\hline Increase in CD4+ count at $48 \mathrm{wk}\left(\mathrm{cells} / \mathrm{mm}^{3}\right)$ & 206 & 206 & \\
\hline Rate of failure after 48 wk (no./100 person-yr) & 1.4 & 1.4 & $0-14.1$ \\
\hline Rate of loss to follow-up (no./100 person-yr) & 3.4 & 3.4 & $0-10.0$ \\
\hline \multicolumn{4}{|l|}{ Transmission } \\
\hline Average no. of secondary partners $/ \mathrm{mo} \S$ & 0.014 & 0.007 & \\
\hline \multicolumn{4}{|l|}{ No. of transmissions/partner/100 person-yr ${ }^{q} /$} \\
\hline Patients with suppression of HIV RNA & 0.10 & 0.10 & \\
\hline Patients without suppression of HIV RNA & 1.48 & 1.48 & \\
\hline Average duration of primary partnership (yr) & 15 & 15 & $5-100$ \\
\hline Average duration of acute infection (mo) & 5 & 5 & $0-12$ \\
\hline Infectivity multiplier for acute infection// & 26 & 26 & $0-50$ \\
\hline \multicolumn{4}{|l|}{ Relative sexual activity according to age ${ }^{* *}$} \\
\hline $15-24 \mathrm{yr}$ & 1.10 & 1.10 & \\
\hline $25-29 \mathrm{yr}$ & 0.99 & 0.99 & \\
\hline $30-34 \mathrm{yr}$ & 1.00 & 1.00 & \\
\hline$>35 \mathrm{yr}$ & 0.82 & 0.82 & \\
\hline \multicolumn{4}{|l|}{ Costs (2011 U.S. \$) } \\
\hline Acute episode of opportunistic disease & $309-1048$ & $43-313$ & Base case $\times 0.25-4.00$ \\
\hline Annual routine care & $293-2390$ & $90-311$ & Base case $\times 0.25-4.00$ \\
\hline CD4+ test & 14 & 6 & Base case $\times 0.50-2.00$ \\
\hline HIV RNA test & 68 & 47 & Base case $\times 0.50-2.00$ \\
\hline \multicolumn{4}{|l|}{ Annual ART } \\
\hline First-line & 153 & 135 & Base case $\times 0.25-1.00$ \\
\hline Second-line & 520 & 561 & Base case $\times 0.25-1.00$ \\
\hline
\end{tabular}

Plus-minus values are means \pm SD. Sources of data are as follows: cohort characteristics, efficacy of first- and second-line antiretroviral therapy (ART), and transmission per partner, ClinicalTrials.gov ${ }^{14}$; partnership duration, Lurie et al. ${ }^{15}$; duration of acute infection, Powers et al. ${ }^{16}$; acute infection infectivity multiplier, Hollingsworth et al. ${ }^{17}$; sexual activity and age, Gray et al. ${ }^{18}$; costs, Médecins sans Frontières and Health Systems Trust $^{19}$ and YRG CARE ${ }^{20}$; and first- and second-line ART cost, World Health Organization. ${ }^{21}$ 
${ }^{\dagger}$ For variables in which between-group differences were significant, characteristics are reported separately for the 954 African participants and the 531 Asian participants; otherwise, values are pooled estimates for the two regions. ART efficacy, rates of loss to follow-up, and transmission rates were calculated for 1763 patients from all sites including those in North and South America. Additional details of the data analysis are provided in the Supplementary Appendix.

For the proportion of patients with HIV RNA suppression at 48 weeks, the $95 \%$ confidence interval was 90 to $94 \%$.

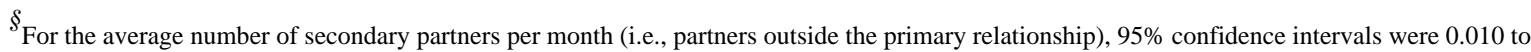
0.019 for South Africa and 0.003 to 0.012 for India.

${ }^{I /}$ For the number of transmissions per partner per 100 person-yr, $95 \%$ confidence intervals were 0.012 to 0.372 for patients with HIV RNA suppression and 0.978 to 2.158 for those without HIV RNA suppression.

"The infectivity multiplier for acute infection was applied to the transmission rate for patients without HIV RNA suppression.

**

Age groups do not include children up to the age of 14 years. The reference group was patients between the ages of 30 and 34 years. 
Table 2

HIV Transmission, Life Expectancy, Costs, and Cost-Effectiveness. ${ }^{*}$

\begin{tabular}{|c|c|c|c|c|c|c|c|}
\hline \multirow[t]{2}{*}{ Variable } & \multicolumn{3}{|c|}{ Index Patients } & \multirow[b]{2}{*}{$\begin{array}{l}\text { Transmissions } \\
\text { per Index } \\
\text { Patient }{ }^{\ddagger} \\
\text { no. }(\% \text { change })\end{array}$} & \multicolumn{2}{|c|}{$\begin{array}{l}\text { Patients with Newly } \\
\text { Transmitted HIV }\end{array}$} & \multirow[t]{2}{*}{$\begin{array}{c}\text { Overall } \\
\text { Incremental } \\
\text { Cost-Effectiveness } \\
\text { Ratio }^{\dagger}\end{array}$} \\
\hline & $\begin{array}{c}\text { Survival } \\
\%\end{array}$ & $\begin{array}{c}\text { Life } \\
\text { Expectancy } \\
\text { mo }\end{array}$ & $\begin{array}{c}\text { Costs } \\
2011 \text { U.S. \$ }\end{array}$ & & $\begin{array}{c}\text { Life-Months } \\
\text { Lost Due to } \\
\text { Transmission } \\
\text { mo }\end{array}$ & 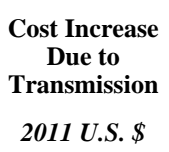 & \\
\hline \multicolumn{8}{|l|}{ South Africa } \\
\hline \multicolumn{8}{|l|}{5 -year period } \\
\hline Delayed ART & 83 & $51.7^{\S}$ & $4,800^{q l}$ & 0.04 & 0.09 & 80 & NA \\
\hline Early ART & 93 & $54.9^{\S}$ & $4,800^{q l}$ & $0.01(-69)$ & 0.02 & 20 & Cost-saving \\
\hline \multicolumn{8}{|l|}{ Lifetime period } \\
\hline Delayed ART & NA & 165.2 & 15,100 & 0.08 & 5.26 & 760 & NA \\
\hline Early ART & NA & 189.4 & 16,600 & $0.07(-13)$ & 2.64 & 560 & 590 \\
\hline \multicolumn{8}{|c|}{ Alternative scenarios } \\
\hline Routine care & & & & & & & $630 / /$ \\
\hline Worst case & & & & & & & $550 / /$ \\
\hline \multicolumn{8}{|l|}{ India } \\
\hline \multicolumn{8}{|l|}{5 -year period } \\
\hline Delayed ART & 87 & $52.8^{\S}$ & 1,800 & 0.04 & 0.06 & 30 & NA \\
\hline Early ART & 94 & $55.1^{\S}$ & 2,200 & $0.01(-66)$ & 0.01 & 10 & 1,800 \\
\hline \multicolumn{8}{|l|}{ Lifetime period } \\
\hline Delayed ART & NA & 182.6 & 7,300 & 0.08 & 3.83 & 370 & NA \\
\hline Early ART & NA & 202.6 & 8,400 & $0.07(-13)$ & 1.95 & 290 & 530 \\
\hline \multicolumn{8}{|c|}{ Alternative scenarios } \\
\hline Routine care & & & & & & & $560 / /$ \\
\hline Worst case & & & & & & & $480 / /$ \\
\hline
\end{tabular}

* NA denotes not applicable.

${ }^{\dagger}$ The overall incremental cost-effectiveness ratio was calculated as the difference in costs for the index patient plus the difference in the increase in costs due to transmission divided by the difference in life expectancy for the index patients plus the difference in life-months lost due to transmission, all discounted at $3 \%$ per year.

FTe percent change in transmissions per index patient is indicated for early ART, as compared with delayed ART, and was calculated before rounding.

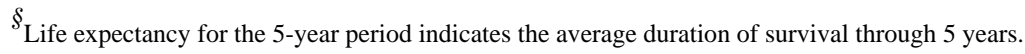

${ }^{I}$ Costs are the same for delayed and early ART because of rounding. Unrounded values are $\$ 4,766$ for delayed ART and $\$ 4,780$ for early ART.

"Additional details of the results of the routine-care and worst-case scenarios are provided in Tables S3 and S4, respectively, in the Supplementary Appendix. 